\title{
Editorial:
}

\section{Marine information technology: the best is yet to come}

\author{
Wen $\mathrm{XU}^{\dagger 1}$, Yuan-liang MA ${ }^{\dagger \star 2}$, Fumin $\mathrm{ZHANG}^{3}$, Daniel ROUSEFF ${ }^{4}$, \\ Fei $\mathrm{JI}^{5}$, Jun-hong CUI ${ }^{6}$, Hussein YAHIA ${ }^{7}$ \\ ${ }^{1}$ College of Information Science and Electronic Engineering, Zhejiang University, Hangzhou 310027, China \\ ${ }^{2}$ School of Marine Science and Technology, Northwestern Polytechnical University, Xi'an 710072, China \\ ${ }^{3}$ School of Electrical and Computer Engineering, Georgia Institute of Technology, Atlanta, GA 30332, USA \\ ${ }^{4}$ Applied Physics Laboratory, University of Washington, Seattle, WA 98105, USA \\ ${ }^{5}$ School of Electronic and Information Engineering, South China University of Technology, Guangzhou 510641, China \\ ${ }^{6}$ Smart Ocean Research Center, Jilin University, Changchun 130012, China \\ ${ }^{7}$ Research Center INRIA Bordeaux - South West, Talence 33405, France \\ †E-mail: wxu@zju.edu.cn; ylma@nwpu.edu.cn
}

https://doi.org/10.1631/FITEE.1820000

Marine information technology (MarineIT) involves marine information gathering, transmission, processing, and fusion. Traditionally, this topic has been referred to in the context of acoustic, optical, and electromagnetic sensing of the ocean environment, most notably sonar/radar processing and satellite remote sensing. As its embodiment becomes enriched and its scope extends, particularly accompanied by the advancements in cabled or wireless ocean observation networks, it is fair to refer to MarineIT as a dedicated discipline of information technologies. MarineIT plays an important role in many applications, such as marine science research, environmental exploration, resource exploitation, and security and defense. Owing to its specific application domain, it has also become a trending topic of the information technology research.

Like other branches of information science, the development of marine information technology over the last 30 years has benefited significantly from advances and achievements in general information theory. However, the manner in which it highlights the close bonding among propagation physics, signal processing, and the marine environment is seldom

\footnotetext{
${ }^{\ddagger}$ Corresponding author

(C) Zhejiang University and Springer-Verlag GmbH Germany, part of Springer Nature 2018
}

seen in other areas (Xu et al., 2016). As such, direct applications of general information methods to ocean environments do not usually work well, and MarineIT presents many features different from its terrestrial counterparts. For example, the ocean volume is seemingly transparent to sound, and thus acoustic waves are used as the main information carrier for underwater sensing and communications. Long-range sound propagation is subject to a so-called waveguide effect, spatially bounded by the sea surface and bottom, and temporally experiencing dramatic variation due to ocean dynamics. While the matched filter concept can still be applied, the signal replica used for matching is no longer a free field solution. Instead, a full-field solution has to be modeled, computed numerically, and even tracked for the given channel. In other words, propagation physical modeling and signal processing should match the ocean environment.

We have witnessed significant progress in MarineIT in recent years due to field-specific developments in signal and information processing, propagation physics modeling, and oceanographic data collection. The use of new observation platforms, such as underwater and surface vehicles, seafloor observatories, and wireless networks, offers important new opportunities. This special issue assembles eight peer-reviewed articles on underwater 
acoustic signal processing and communication, optical image processing, remote sensing, and application of unmanned underwater vehicles. This effort is intended to enlighten the research community about the recent progress in the field of MarineIT. It may also offer some insights into identifying important scientific issues to be addressed in the future.

\section{Paper overview}

Underwater acoustic communication (UAC) has recently attracted much attention in the fields of both acoustics and wireless communications, owing to the unique challenges of the underwater channel. These include spreading in both time delay and Doppler, a highly time-varying environment, non-line-of-sight propagation paths, and scattering from the dynamic sea surface. Huang et al. (2018) discussed in detail some of the difficulties a UAC system faces, and presented an overview of research results from the physical layer to the network layer, with emphasis on high data rate modulation schemes for short- and medium-range UAC, namely single-carrier timedomain equalization (SC-TDE), single-carrier frequency-domain equalization (SC-FDE), orthogonal frequency division multiplexing (OFDM), and the relatively new orthogonal signal division multiplexing (OSDM). They also proposed a so-called "PIEs" criterion as a tool to evaluate general performance and assist the design of a UAC system from the perspective of system designers. Experimental results of short- and medium-range UAC systems with high data rates after the year 2000 were summarized to accompany the proposed UAC general performance evaluation criteria.

Liu et al. (2018) developed a UAC scheme based on direct sequence spread spectrum with potential network applications. To solve the contradiction between the information data rate and the accuracy of Doppler estimation, channel estimation and frame synchronization, a data frame structure based on dual spread spectrum code was designed, with long spread spectrum code used as the training sequence and a short counterpart used to modulate the message. Simulations were implemented to verify performance at a low signal-to-noise ratio (SNR).

Underwater video transmission is of increasing interest given the success in sea trials of the Chinese manned submersible "Jiaolong." Current designs often treat video coding and transmission schemes separately. Zhang et al. (2018) proposed an adaptive transceiver by considering the importance of both video components and channel conditions. Both precoder and equalizer design were derived for the respective perfect and imperfect channel state information (CSI) cases. The simulation results revealed that the new design is effective in terms of better peak SNR and higher video structural similarity index, and is also resistant to channel estimation error.

Underwater wireless sensor networks (UWSNs), as an extension of their terrestrial counterpart, have been intensively studied in the past 10 years, with potential applications in environmental sensing and target tracking. In addition to communication, node localization is an important issue to be addressed, particularly considering that an underwater node may move around with the currents and the lack of GPS. Chen et al. (2018) proposed a high-precision localization algorithm with mobility prediction. They also presented a simultaneous localization and target tracking algorithm to update sensor locations based on measurements made during the process of target tracking. Energy efficiency of the developed algorithm was justified using the simulation data.

Underwater acoustics is essentially an observational science, and research on UWSNs has been criticized principally for the lack of meaningful experiments. The remarkable recent advances in unmanned underwater and surface vehicles, however, present an opportunity to answer these criticisms by implementing UWSNs in the field. Movements of the vehicles equipped with information devices generate streams of information/data, revolutionizing the stateof-the-art in ocean observations. Tao et al. (2018) presented the integration of a compact acoustic communication device with a micro-sized autonomous underwater vehicle (AUV). Packet reception rate and bit error rate of the acoustic communication link were evaluated in a confined pool environment, using experiments in lake environments as a comparison. Several major factors, including multi-path, path hopping, and Doppler effects, were recognized as significantly impacting mobile communication performance.

Endurance time and navigation accuracy are two limiting factors in AUV applications. Underwater 
docking greatly facilitates and extends operation of an AUV, thus being of significant interest to both the research and application communities. Li et al. (2018) introduced a docking system with an under-actuated AUV, which requires both highly accurate cross-track and heading controls to ensure successful docking operations. Considering that the presence of unknown currents is common in practical scenarios, they focused on the design and implementation of the docking strategy, controller, current estimator, and compensator. A series of sea trial results were presented to validate the feasibility and effectiveness of the proposed control strategy as well as current estimator and compensator, thus distinguishing this paper from others on the same topic. Notably, it demonstrated the robustness of the docking system as a whole.

Tan et al. (2018) analyzed deep water video sequences acquired by a remotely operated vehicle (ROV). Aimed at facilitating Norway lobster stock assessment, they proposed an automated video analysis framework to detect, track, and quantify both this particular benthic species and its biogenic feature (the main burrow's entrance) using properly handled ROV dynamic motion. Encouraging results were obtained for the available test videos when comparing the manual counts by human experts against the automatic video-based abundance estimation. In spite of these findings, it was stated that the amount of missed and misidentified burrows may represent a hindrance for the immediate adoption of this technique in field operations.

Yahia et al. (2018) presented an experiment, conducted over one year of daily remotely sensed data collection, to discover different turbulent statistics of the oceanic system, the goal being to study the wind stress forcing effect on ocean dynamics at the air-sea interface. The study was conducted by computing statistical moments on the shapes of spectra within the framework of microcanonical multifractal formalism. Properties of singularity spectra, computed from velocity norms and vorticity data, were explored to underline differences in turbulent regimes associated with both velocity fields of geostrophy with and without wind stress forcing. We should also mention that although this paper is the only representative in the collection of studies on remote sensing processing, the topic certainly constitutes one of the main areas in marine information research.

\section{Scientific rationale and outlook}

As the showcased articles show, a typical marine information problem addresses observation, communication, or control. The theoretical foundation comprises informatics, with both information theory and estimation theory as the core. Information and physics constitute the world (space) we inhabit. Hence, marine information research is often undertaken to discover the relationship between information, physics, and space. The physical problem of how this information is carried and spreaded can boil down to propagation physics. The intersection of information and space is where the signal lies. Communication, observation, and control are all to extract the information embedded in signals, and guarantee the estimability, detectability, stabilizability, controllability, and observability of the physical world (Xu et al., 2016).

A framework of information, physics, and space is illustrated in Fig. 1, which completely describes the natural world from the perspectives of both physics and informatics (Xu et al., 2016). The estimation theory, Hilbert space/operator theory, and information theory are three basic elements; interactions of these

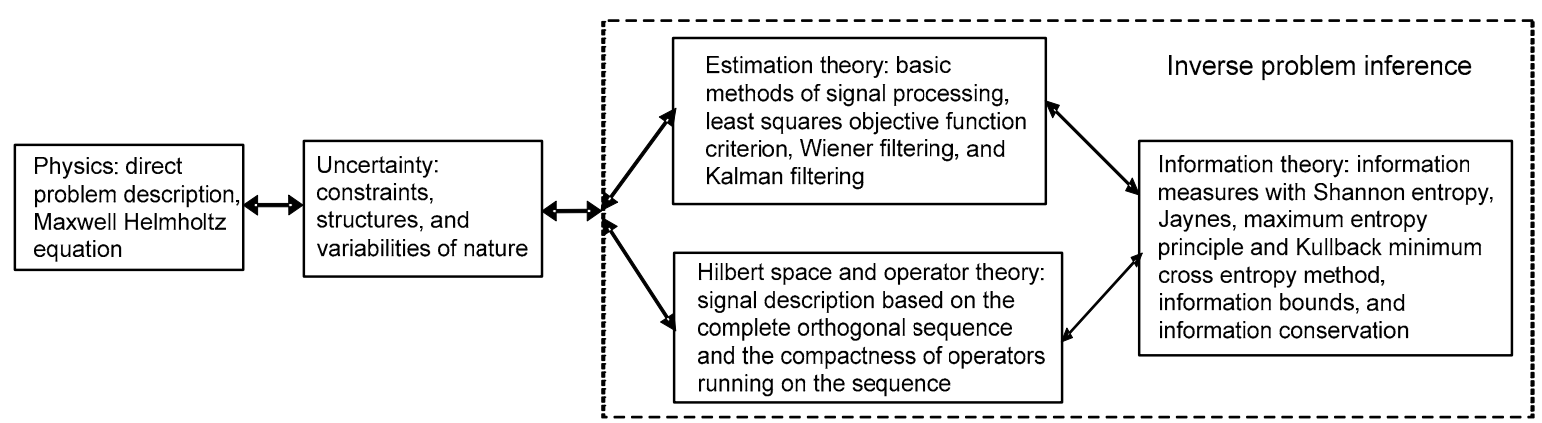

Fig. 1 Relationship between information, physics, and the Hilbert space (Reprinted from Xu et al. (2016), Copyright 2016, with permission from Science China Press) 
three elements produce theoretical bounds for various inverse/inference problems and basic signal processing methods. This framework also unifies both observation and communication. O'Sullivan et al. (1998) systematically presented the model and methodologies of information-theoretic image formation for observation. The source, encoder, channel, and decoder in a communication model were replaced with source, sensors, and imager for observation. Even though the theory of information-theoretic imaging is far from mature, it has the potential of playing as powerful a role in imaging as Shannon's source-channel model plays for communications.

The above framework embodies many research studies in the past, and, we believe that it will certainly motivate a lot more for the future, with the best yet to come. Some example topics for future study include the following (Xu et al., 2016):

1. Physical mechanisms for probing the ocean using acoustic, optical, and electromagnetic methods and their coupling with the environment: Recent research has graduated from static to dynamic environmental modeling, the aim being to capture the dynamic coupling between ocean processes and propagation physics. In this manner, remote sensing may also extend to deep underwater environments. New signal processing and data assimilation methods can thus be developed based on dynamic coupling.

2. Large-scale general underwater imaging and scenario analysis: Ocean observation is typically presented as an under-determined inverse problem, and imaging is one of the effective solutions.

3. Underwater and transmedia communications: Topics include adaptive modulation, duplexing acoustic communication, channel modeling and identification, network resource dynamic scheduling with strong constraints, and communication across the air-sea interface.

4. Autonomy of marine vehicles for ocean sensing: Cyber maritime cycles are key to autonomy of marine vehicles. Recent research trends favor the development of distributed cycles so that information can flow smoothly among vehicles. This would allow more flexible data streams and transforms (e.g., using a swarm of vehicles to map underwater acoustic fields, or collecting data from underwater sensor networks).

5. Intelligent data processing and ocean big data: Marine data processing faces data of massive volumes, from multiple sources, and spanning multiply scales, dimensions, and modes. Intelligent processing involves multi-source heterogeneous data fusion, data mining and analysis, numerical simulation, super computation, and visualization. Big data methodologies may be applied to process at least massive data from remote sensing or Argo floats.

Last, but certainly not the least, we appreciate all the authors, reviewers, and staff editors for their participation and assistance, without which this special issue would not come to shape. Special thanks are offered to Prof. Xianyi GONG for his insightful thoughts on the relationship between information, physics, and space.

\section{References}

Chen HY, Liu MQ, Zhang SL, 2018. Energy-efficient localization and target tracking via underwater mobile sensor networks. Front Inform Technol Electron Eng, 19(8): 999-1012. https://doi.org/10.1631/FITEE.1700598

Huang JG, Wang H, He CB, et al., 2018. Underwater acoustic communication and the general performance evaluation criteria. Front Inform Technol Electron Eng, 19(8):951971. https://doi.org/10.1631/FITEE.1700775

Li B, Xu YX, Fan SS, et al., 2018. Underwater docking of an under-actuated autonomous underwater vehicle: system design and control implementation. Front Inform Technol Electron Eng, 19(8):1024-1041. https://doi.org/10.1631/FITEE.1700382

Liu LJ, Li JF, Zhou L, et al., 2018. An underwater acoustic direct sequence spread spectrum communication system using dual spread spectrum code. Front Inform Technol Electron Eng, 19(8):972-983. https://doi.org/10.1631/FITEE.1700746

Tan CS, Lau PY, Correia PL, et al., 2018. Automatic analysis of deep-water remotely operated vehicle footage for estimation of Norway lobster abundance. Front Inform Technol Electron Eng, 19(8):1042-1055. https://doi.org/10.1631/FITEE.1700720

Tao QY, Zhou YH, Tong F, et al., 2018. Evaluating acoustic communication performance of micro autonomous underwater vehicles in confined space. Front Inform Technol Electron Eng, 19(8):1013-1023. https://doi.org/10.1631/FITEE.1700841

O'Sullivan JA, Blahut RE, Snyder DL, 1998. Informationtheoretic image formation. IEEE Trans Inform Theory, 44: 2049-2123.

Xu W, Yan S, Ji F, et al., 2016. Marine information gathering, transmission, processing, and fusion: current status and future trends. Sci Sin Inform, 46(8):1053-1085 (in Chinese). https://doi.org/10.1360/N112016-00064

Yahia H, Garcon V, Sudre J, et al., 2018. Effect of wind stress forcing on ocean dynamics at air-sea interface. Front Inform Technol Electron Eng, 19(8):1056-1062. https://doi.org/10.1631/FITEE.1700797

Zhang RX, Ma XL, Wang DQ, et al., 2018. Underwater video transceiver designs based on channel state information and video content. Front Inform Technol Electron Eng, 19(8):984-998. https://doi.org/10.1631/FITEE.1700767 